\title{
Über die Ursache der Maculae coeruleae.
}

\author{
Von
}

\author{
Privatdozent Dr. MY. Oppenheim.
}

(Hiezu Taf. III.)

Im Band XCI des Archivs für Dermatologie und Syphilis beschäftigt sich Ti èch e mit der Pathogenese der Tâches bleues, ohne jedoch zu einer wirklichen Klärung der Frage zu kommen. Er beobachtete an frisch gefangenen Morpionen Klümpchen von menschlichem Blute, die sich unter dem Mikroskope als braune und dunkelblaue schollige Massen präsentierten. Auch fand er bei vernachlässigten Morpionenträgern kleine braunrote Krüstchen, in denen sich neben den oben erwähnten Schollen auch lila Körnchen befanden, deren Farbe an die Tâches bleues erinnerte. Im Anschlusse an diese Beobachtung gab Tièche menschliches Blut und zerschnittene Morpionen zusammen und sah dabei unter dem Mikroskope eine Violettfärbung an den roten Blutkörperchen, die er als eine Reduktion des 0xyhämoglobins durch überlebendes Gewebe auffaßt; auch beim Zusammengeben von menschlicher Haut mit menschlichem Blute fand or diese Verfärbung, wenn auch in geringem Grade. Man könne daher, meint Tièche, möglicherweise die Entstehung der Tâches bleues so erklären, daß das durch den Biß des Tieres unter die Haut ausgetretene Blut durch letztere reduziert werde; anderseits sei die Hypothese nicht notwendig, da auch reines Rot durch die Haut als trübes :Medium angesehen bläulich erscheint. 


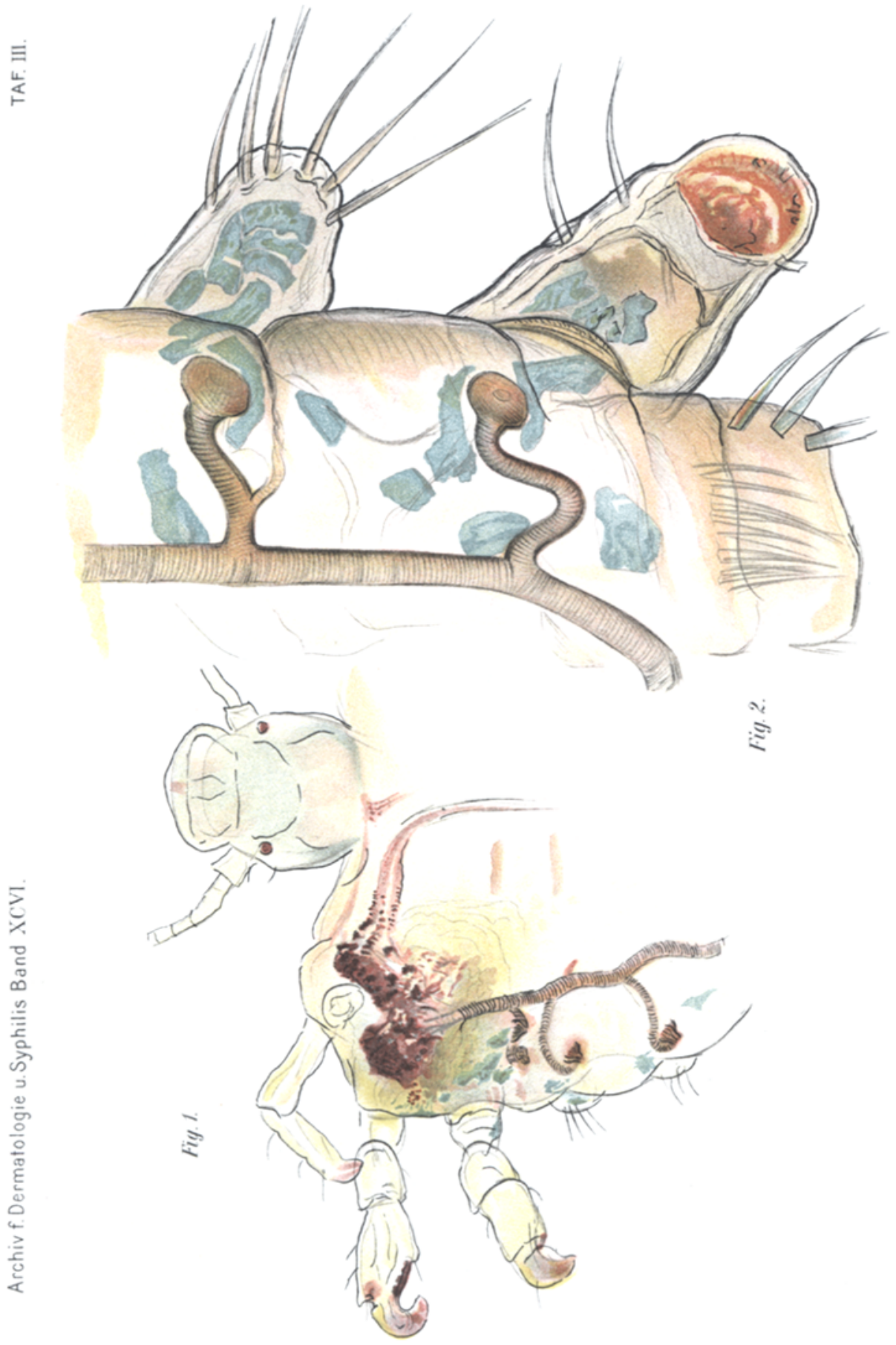

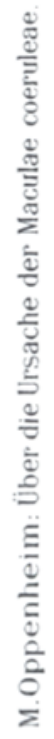


Ferner untersuchte Tièche die Maculae coeruleae auf eine neue Art, da bisher die histologische Untersuchung resultatlos geblieben war. Er trug wie bei einer Thierschschen Transplantation die oberen Hautschichten über Tâches bleues ab und konnte in dem abgeschnittenen Läppchen nichts von Färbung konstatieren, wohl aber an der zurückgebliebenen Haut nach Stillung der Blutung, Daraus schließt er, daß es sich um eine Infiltration der Haut mit korpuskulären Elementen handelt, die sehr fein und unterhalb des Epithels gelegen sein müssen. An eine Imbibition mit gelöstem Marerial sei weniger zu denken, weil das lange Bestehen der Tâches bleues dagegen spräche. Das färbende Material stammt aus dem Blute, ohne daß jedoch zu dessen Entstehung ein besonderes Ferment notwendig wäre, da so geringe Mengen Blutfarbstoff wohl kaum sonst mit der menschlichen Haut ohne Entzündungserscheinungen in Berührung kommen.

Ferner beschäftigt sich $\mathrm{Ti}$ èche mit der Frage des Retraktärsein gewisser Menschen gegenüber der Entwicklung von Tâches bleues und kommt auch hier auf Grund seiner Beobachtungen und Experimente zu einem von den anderen Autoren abweichenden Resultate, indem er das Refraktärsein, wenn es überhaupt vorkommt, als sehr selten hinstellt. Das spärliche Vorkommen der Tâches sei durch eine trockene, ichthyotische, spröde und abschilfernde Haut bedingt, während Menschen mit zarter feiner Haut und solche, die stark schwitzen, mit leicht erregbarem Nervensystem leicht Maculae bekommen. Manchmal sei die geringe Entwicklung der Maculae dadurch begründet, daß die Phthirii krank sind und langsam absterben, bevor es zur wirklichen Bildung der Tâches kommen konnte.

Die Basis für die Meinungen des genannten Autors bilden demnach die Veränderungen des menschlichen Blutes durch Morpionen und überlebendes Gewebe, die blaue Farbe, die die Wundfläche nach Entfernung Thiers ch scher Läppchen von Tâches bleues zeigt und die künstliche Erzeugung von Tâches auch bei scheinbar refraktären Menschen. Mikroskopisch wurden weder die Pedikuli noch die Tâches bleues untersucht.

Was den ersten Punkt betrifft, so findet man die eingangs erwähnten braunen und dunkelblauen Schollen und die lila 
Körnchen bei allen Pedikulösen. Menschen, die Pediculi capitis oder Pediculi restimentorum haben, zeigen dieselben Gebilde, die ja nichts anderes sind als Reste eingetrockneten und veränderten Blutes, das entweder an der Stichstelle eingetrocknet war oder am Schnabel des Tieres geklebt oder als verdautes Blut, den Darmkanal des Tieres passiert hatte. Wenn man die verschiedenen menschlichen Pedikuliarten lebend unter dem Mikroskope untersucht, so kann man öfters an den Mund- und Afteröffnungen, sowie an den Chitinstacheln des Körpers, an den Extremitäten und Antennen derartige Schollen und Körnchen antreffen.

(Eine ähaliche Beobachtung beschreibt Campos Huguenay, der einen roten Staub, Poussiere rougeâtre beschreibt, den er bei disponierten Morpionenträgern beobachtet hat und dessen Auftreten mit der Entwick. lung der Tâches bleues in Zusammenhang gebracht wird.)

Es kommen also diese Schollen und Körnchen nicht nur bei mit Morpionen behafteten Menschen allein vor, sondern auch bei anderen Pedikulösen, während die Tâches bleues ausschlieblich durch die Anwesenheit von Peảiculi pubis hervorgerufen sind, woran heutzutage niemand mehr zweifelt. Schon aus dieser Erwägung allein heraus dürfen sie nicht zur Erklärung der Tâches bleues herangezogen werden, da ein direkter Zusammenhang zwischen der Erzeugung von Tâches bleues und diesen Körnchen nicht bestehen kann.

Hingegen möchte ich an dieser Stelle auf eine andere Beobachtung hinweisen, die sich bei der mikroskopischen Untersuchung von lebenden und toten, mit Alkohol und Xylol zum Zwecke des Einschlusses in Kanadabalsam behandelten Morpionen ergibt. In meiner ersten Publikation: „Über einen durch den Pediculus pubis erzeugten Farbstoff" habe ich Zellen beschrieben, auf die ich von Prof. Ehrmann aufmerksam gemacht wurde, die dem Fettkörper (Mesenterium) des Tieres angehören, leuchtend grüne, verschieden große Körnchen enthalten, und gewisse Reaktionen zeigen. Auf Grund dieser Reaktionen erschien der Schluß berechtigt, daß diese grünen Körnchen aus dem Hämoglobin des menschlichen Blutes durch Oxydation entstünden, ähnlich dem Biliverdin, von dem man dasselbe annimmt. Die Oxydation würde durch ein Ferment (Oxydase) vermittelt, das die großen Speicheldrüsen des Pedikulus ent- 
halten; in der menschlichen Haut spiele sich infolge des Bisses des Morpio ein ähnlicher Vorgang $a b$; der grüne Farbstoff erscheine, durch die Haut als trübes Medium angesehen, blau. Wenn man viele Morpiones untersucht, so findet man Exemplare, die grüne Körnchen in Zellen und frei liegend symmetrisch zu beiden Seiten des Saugrüssels, dort, wo die Speicheldrüsen sind, dann in der Nähe der Mund- und Afteröffnung zeigen. Bei meinen ersten Untersuchungen konnte ich sie an diesen Stellen nicht finden. Hat man Exemplare, die sich eben mit Blut vollgesogen haben, so sieht man im Y-förmigen Magen des Tieres noch die einzelnen Blutkörperchen ziemlich erhalten; in toto erscheint der Inhalt des Magens intensiv rot. In den distalen, Y-förmigen Ausläufern, die sich bis in die Wurzeln des ersten Extremitätenpaares erstrecken, wird die Färbung mehr dunkelbraunrot und hier sieht man auch stellenweise blau gefärbte Partien. Diese blaue Färbung des Mageninhaltes ist nicht nur bei lebenden Pedikuli zu sehen sondern auch bei seit ]ängerer Zeit abgestorbenen, wofern deren Magen mit Blut gefüllt war. Weniger deutlich erscheint sie in den Dauerpräparaten, wo der Pedikulus nach Behandlung mit absoluten Alkohol und Xylol in Kanadabalsam eingeschlossen ist; doch kann man auch hier bläuliche Färbungen speziell an den Rändern des Mageninhaltes erkennen.

Diese blauen Farbentöne entstehen offenbar durch Veränderungen des Blutes im Magen; ob sie nur beim Pediculus pubis vorkommen oder auch bei anderen Arten, konnte nicht entschieden werden, da ich zu wenig andere Arten untersucht habe.

Weit wichtiger jedoch für die Frage der Herkunft der ausschließlich bei den Morpionen vorkommenden grünen Farbe schien folgendes zu sein: In manchen Abdominalzapfen von Tieren, deren Magen Blut enthielt und die reichlichst grüngefärbte Zellen hatten, sieht man in unmittelbarer Nachbarschaft der grünkörnigen Zellen, solche, welche rote und rotbraune, auch gelbe und gelbbraune Körner enthalten, deren Farbe den im Darmkanal und im Magen befindlichen veränderten Biute entspricht. Man findet also grüne, rote und gelbe Zellen unmittelbar nebeneinander in einem Abdominalzapfen. Und das beobachtete ich häufig; seltener konnte man 
konstatieren, daß der braunrote Mageninhalt in den oben beschriebenen seitlichen Magenteilen, gegen das Abdominalende des Tieres allmählich mehr gelbe dann gelbgrïne Farbennuancen zeigte. Im unmittelbaren Anschlusse an eine gelbgrün gefärbte Partie fand ich dann in der Nähe der ersten Thoraxtrachee die typischen leuchtend grünen Zellen. (S. Tafel.)

Auch kann man an Dauerpräparaten die Beobachtung machen, daß die grüne Farbennuance der Fettkörperzellen sich ändert. In der ersten Zeit haben die grïnen Körnchen der farbstoffhaltigen Zellen einen Ton, der mehr ins bläuliche geht, später verliert sich dieser und die Farbe wird leuchtend smaragdgrün. So bleibt sie dann konstant; die ersten Präparate, die ich vor 7 Jahren machte, zeigen die grüne Farbe in den Zellen noch ebenso leuchtend und intensiv, wie zur Zeit meiner ersten Publikation.

Aus allen diesen Beobachtungen geht wohl hervor, daß die grüne Farbe der Fettkörperzellen im Morpionenleibe aus dem Hämoglobin des menschlichen Blutes stammt und daß dieses durch gewisse Umsetzungen, zu denen wir genug Analogien besitzen, die Farbenskala rom Rot über das Rotbraun und Blau zum Blaugrün und Grün durchläuft.

Gegen die Annahme Ti èches, daß die Maculae coeruleae durch minimale Blutaustritte entstehen, wobei das Blut entweder durch die Haut reduziert wird oder auch als solches den Tâche hervorruft, sprechen folgende Erwägungen: Es gelingt niemals durch einen Stich in die Haut, auch wenn dieser mit noch so feinen Instrumenten ausgefiuhrt wird, durch den ihm folgenden Blutaustritt allein, eine Maculae coerulae zu erzeugen. Andere stechende und saugende Insekten wie Morpiones, die nicht immer durch den Stich oder Biß Entzündung erregen müssen, erzeugen niemals Tâches bleues, obwohl die Bedingungen zum Zustandekommen der Tâche nach Tièche dabei völlig erfüllt sind. Duguet, Mallet sowie uns und anderen Autoren ist es gelungen, durch Extrakt von verriebenen Morpionen und durch den Detritus dieser selbst bei Einbringung derselben unter die Haut mittelst eines Stiches Tâches bleues zu erzeugen; mit Extrakten von Kopf- und Kleiderläusen und mit deren Detritus gelingt dies nicht. Aus diesen Experimenten 
folgt unmittelbar, daß es nicht das Blut alle in und auch nicht dessen Veränderung $\mathrm{d} u \mathbf{r} \mathrm{ch}$ die Haut sein kann, die die Tâche bleue erzeugen, sondern daß dazu der Pediculus pubis oder dessen Teile unbedingt notwendig sind. Wäre dies nicht der Fall, so könnte ja die Macula coerulea nicht nur durch den Pediculus pubis allein bedingt sein.

Was die Untersuchung der Tâches bleues selbst betrifft, so waren die histologischen Forschungen bisher nach den übereinstimmenden Angaben aller, die sich mit dieser Frage beschäftigt haben, negativ. Es konnten weder Färbungen noch pathologische Änderungen in den Bestandteilen der Cutis und Epidermis konstatiert werden. Wie ich in der Abhandlung über die "Maculae coeruleae" im Handbuche für Hautkrankheiten von Mraček schrieb waren auch meine diesbezüglichen zahlreichen histologischen Untersuchungen mit verschiedenen Fixierungs-, Härtungs- und Färbungsmethoden resultatlos geblieben. Ti ì ch $\mathrm{e}$ fand nun, wie eingangs erwähnt, nach Abtragung $\mathrm{T}$ h i e r s c h scher Läppchen, die blaue Farbe nur an dem Cutisteil, nicht aber an dem Läppchen und schließt daraus auf die Bildung der Farbe unterhalb des Epithels und Papillarkörpers. Dies würde mit dem Experiment Ciuffos übereinstimmen, der nur nach subkutaner Einverleibung eines Pedikuli-Extraktes Tâches bleues hervorrufen konnte, nicht aber bei oberflächlicher Skarifikation.

Meine Untersuchungen der Maculae coeruleae die ich nun von Neuem aufnahm, ergaben von dem eben Angeführten ein wenig abweichende Resultate.

Exzidiert man eine Tâche bleue, ${ }^{1}$ ) so sieht man, daß auch das subkutane Gewebe, von dem man die Tâche losgelöst hat, einen deutlichen blauen Farbenton zeigt. Durchschneidet man das exzidierte Hautstïck, so sieht man die Schnittfläche deutlich blau gefärbt und zwar betrifft die Blaufärbung die ganze Schnittfläche, sie reicht vom Epithel bis ins subkutane Fettgewebe.

Die Schnittflächen behalten die blaue Farbe an der Luft durch längere Zeit; auch im Wasser kann man nach 24 Stunden noch eine bläuliche Färbung wahrnehmen; in Alkohol jedoch

1) Herr Professor Ehrmann hatte die Güte mir das Material seiner Abteilung zur Verfügung zu stellen, wofür ich ihm herzlichst danke. 
schwindet die blaue Farbe sehr bald. Gefrierschnitte, die man mit Chloräthylspray anfertigt, zeigen bei der mikroskopischen Untersuchung im durchfallenden Lichte sowohl als gefärbte als auch als ungefärbte Präparate keinerlei charakteristische Merkmale; untersucht man jedoch Gefrierschnitte, die im Wasser aufgefangen wurden, im auffallenden Lichte, so tritt ein ausgesprochener bläulicher Farbenton hervor, der verschiedene Intensität an verschiedenen Stellen des Präparates hat und im Epithel ebenso anzutreffen ist wie im Bindegewebe.

An gehärteten Präparaten von Maculae coeruleae sieht man eine blaue Farbe, wenn man als Härtungs- und Fixierungsmittel Aceton wählt und die Paraffinschnitte auf Wasser über dunklem Grund in der Aufsicht betrachtet. Man gibt die Stücke unmittelbar nach der Exzision in Aceton, läßt sie daselbst 1-2 Stunden, hierauf kommen sie in Xylol und Paraffin, worin sie dann eingebettet werden. Allein diese blaue Färbung zeigen auch andere nach obigem Verfahren hergestellte Paraffinschnitte. Durch die Acetonhärtung erhalten die Präparate einen blauen Farbenton, der nur im Wasser sichtbar ist; an getrockneten oder nach den üblichen Methoden mit Xylol, Alkohol, Celloidin und Alkohol vorbehandelten Paraffinschnitten sieht man nichts mehr von blauer Farbe. Auch auf andere Weise gehärtete Maculae coeruleae zeigen nichts charakteristisches; es ist also die bei Acetonhärtung auftretende blaue Farbe keine Eigenschaft der Maculae coeruleae, sondern offenbar als Farbe trüber Medien aufzufassen, die durch die Schnellhärtung mit Aceton bedingt wird.

In den zahlreichen Präparaten von Maculae coeruleae, die ich mikroskopisch untersuchte, konnte ich nie die Anwesenheit von Körnchen feststellen, die die Färbung derselben hätte erklären können, weder in den mit Aceton noch in den mit anderen Methoden fixierten und gehärteten Präparaten, wuch nicht bei stärkster Vergrößerung. Es ist daher wohl der Schluß zulässig, daß die blaue Färbung der Tâches bleues durch diffuse Färbung der Haut in allen Teilen zustande kommt. Auch Tièche meint, daß man eventuell an eine Imbibition der Haut mit gelöstem Materiale denken könne, doch spreche das lange Bestehen der Färbung dagegen. 
Was die Frage des Refraktärseins gewisser Menschen in Bezug auf die Entstehung der Tâches bleues anlangt, so habe ich die Experimente Tièches nicht nachgeprüft. Er konnte auch bei scheinbar refraktären Menschen experimentell Tâches bleues erzeugen, indem er ein mit einem kleinen Fenster versehenes Stïck Zinkoxydpflastermull auf die Haut klebte, dann lebende, nicht lädierte Morpionenj in die Fensteröffnung brachte und das ganze dann mit einem Zinkoxydpflaster bedeckte, das an der Stelle, wo es über den Morpionen lag, zur Verhütung der Anklebung etwas Mosetigbatist trug. Auf diese Weise gelang es $\mathrm{T}$ i è $\mathrm{ch}$ e immer Maculae coeruleae zu erzeugen; er konnte auch in Übereinstimmung mit diesen Beobachtungen bei 70 untersuchten, mit Morpionen behafteten Leuten nur ein einzigesmal die dauernde Abwesenheit von Maculae konstatieren und fuihrt dies in diesem einzigen Falle auf Krankheit der Pedikuli zurück, die entweder von vorneherein krank waren oder durch das ihnen nicht zusagende Terrain der an Retinitis albuminurica erkrankten Patientin zu Grunde gingen.

Von einem absoluten Refraktärsein habe ich in meinen Publikationen nicht gesprochen; ich führte die geringe Entwicklung oder das Fehlen der Tâches bleues trotz reichlicher Morpionenentwicklung einerseits darauf zurück, daß vielleicht das Blut gewisser Menschen mit dem rom Pedikulus stammenden Ferment den grünen Farbstoff nicht gibt, anderseits auf den Umstand, daß vielleicht gewisse Pedikulusstämme den Farbstoff überhaupt nicht bilden, da man Pedikuli ohne Farbstoff häufiger bei Menschen antrifft, die spärliche oder keine Tâches bleues haben. Dies stimmt eigentlich mit der Erklärung Tièch es für seinen einzigen Fall von fehlenden Tâches bleues, bei dem er sie doch dann experimentell durch lebende Morpionen erzeugen konnte - kranker Pedikulusstamm, - verändertes nicht zusagendes Terrain - überein. In Bezug auf die klinische Beobachtung, daß Menschen mit zarter, feiner Haut und blonde Individuen reichlicher und leichter Maculae bekommen, als solche mit trockener spröder Haut und brünette Leute sind wir und ebenfalls andere Autoren einer Meinung. Auch bezüglich des Einflusses des Schwitzens und es fragt sich nur ob bei den Experimenten Tièches nicht durch den Mosetig- 
battist und das Zinkoxydpflaster, die die Feuchtigkeit der Haut zurückhalten, günstig auf die Bildung der Maculae eingewirkt wurde. Es wäre die Möglichkeit zu erwägen durch künstliches Schwitzen und dadurch erzeugte Auflockerung und Durchtränkung der Epidermis, die Entwicklung von Maculae coeruleae bei Morpionenträgern zu fördern.

Wir können uns daher der Meinung Tièches, daß die Tâches bleues durch minimale Blutaustritte, die entweder durch die Haut reduziert oder unverändert als feiner, körniger Farbstoff der tieferen Cutisschichten durch die Haut als trübes Medium angesehen die Maculae coeruleae erzeugen, nicht anschließen.

Unsere Ansicht, die wir in den früheren Publikationen entwickelt haben, müssen wir aufrecht erhalten, nur in der Hinsicht ein wenig modifiziert daß wir statt eines körnigen einen gelösten Farbstoff für wahrscheinlicher annehmen, der das Hautgewebe in toto imbibiert. Es lautet daher unsere Erklärung für die Maculae coeruleae folgendermaßen:

Die Maculaecoeruleae, die ausschließlichdurch den BiB des Pediculus pubis entstehen, werden durch einen grünen, gelösten Farbstoff erzeugt, der alle Hautschichten in diffuser Weise färbt. Der Farbstoff entsteht aus dem menschlichen Blute, durch die Sekrete des Tieres, die das Blut auch im Inneren des Tieres in einen grünen, nur dem Pediculus pubis zukommenden Farbstoff umandeln.

\section{Nachtrag.}

In Nr. 2 des XLVIII. Bandes der Monatshefte für prakt. Dermatologie sind inzwischen von Pellier blaue Körner in den Pedikuluseiern beschrieben worden, woraus P. schließt, daß der Pediculus pubis imstande sei, einen blauen Farbstoff direkt, ohne menschliches Blut zu sezernieren. Im Tier selbst hat P. 
weder grüne noch blaue Körner gesehen. Ohne auf die Publikation bier näher eingehen zu wollen, sei nur bemerkt, daß es mir weder jetzt von neuem, noch bei meinen früheren Untersuchungen gelungen ist, in den Eiern irgendwelchen färbigen Bestandteil nachzuweisen. An durchsichtigen und durchscheinenden Chilinstellen sieht man bei wechselnder Lichtrichtung oft Interferenzerscheinungen den Spektralfarben entsprechend; vielleicht hat Pellier da blaue Färbungen gesehen. Daß er die grünen Körnchen in den Pedikulis nicht gesehen hat, kann ich mir nicht erklären. Nur Embryonen und eben ausgekrochene Pedikuli zeigen keinen Farbstoff; die ausgewachsenen zum allergrößten Teil. Auch dieser Umstand spricht für die von uns gegebene Erklärung.

Die Erklärung der Abbildungen auf Taf. III ist dem Texte zu entnehmen. 